\title{
Environmental Perception for Information and Immune Control Algorithm of Miniature Intelligent Vehicle
}

\author{
Yanting Lan ${ }^{1}$, Jinying Huang ${ }^{2}$ and Xiaodong Chen ${ }^{3}$ \\ ${ }^{1}$ North University of China, School of Computer Science and Control \\ Engineering, Taiyuan, 030051, China \\ ${ }^{2}$ North University of China, School of Mechanical and Power Engineering, \\ Taiyuan, 030051, China \\ ${ }^{3}$ Key Laboratory of Farming System, Ministry of Agriculture of the People' $s$ \\ Republic of China, College of Agronomy and Biotechnology, China Agricultural \\ University, Beijing 100193, China \\ 1'lytcyb@foxmail.com, ${ }^{2}$ jyhuang@nuc.edu.cn, ${ }^{3}$ xiaodong136@foxmail.com
}

\begin{abstract}
This paper proposes a simulation platform for vehicle steering using MATLAB based on vehicle dynamics simulation software CarSim to verify the immune control of algorithm performance in order to check the match between the algorithm designed to test the intelligent vehicle immune control and the external sensor through a typical design of simulation. The simulation results show that the proposed algorithm is robust and adaptable to road tracking accuracy of the immune control algorithm and external variable environment and vehicle speed.
\end{abstract}

Keywords: intelligent vehicle, data fusion, immune control, OMAP

\section{Introduction}

Intelligent vehicle is partly or totally to replace driver of the vehicle, by monitoring condition of the driver, road environment and vehicle's own status with systems of advanced vehicle sensing, information processing, decision-making and implementation, and to complete the driving operation. Thereby, the vehicle reduces driver's labor, and improves traffic safety and efficiency, energy conservation and so on. Intelligent vehicle and all kinds of products developed on their basis have become popular to be the key equipment of automation logistics transportation for flexible production enterprises. Nowadays, many developed countries are carrying on the research, design, development and application of intelligent vehicle products. Even, application of intelligent vehicles has been expanding from simply indoor to outdoor, water, air and outer space of a variety of complex situations, in various aspects of military, civil and scientific research[1,2].

According to characteristics of real vehicle tests for long development cycle, large funds, quality of experimental equipment and test personnel, the test in this experiment has utilized a model vehicle, in 1/10 size of a real one(as shown in Figure 1), on the road of campus, Test speed $1-2 \mathrm{~m} / \mathrm{s}$ of the model corresponds to original scale about $30 \sim$ $60 \mathrm{~km} / \mathrm{h}$. Video data of condition in front of the vehicle is collected by communication of a camera connected with USB interface, and inertial navigation information of the vehicle is acquired by communication of a gyroscope module with UART interface. Information acquisition of obstacles around vehicle and artificial control signal are achieved by arrangement of several ultrasonic modules around the vehicle, design and production of a collector to collect the detected obstacle information with ultrasonic module and remote control receiver signal, uploaded through the UART interface on OMAP board. Autonomous navigation, automatic obstacle avoidance and other functions are realized by 
design and production of a controller to receive control information through UART interface on the OMAP board and to send control signals to the motor and the steering gear of the vehicle.

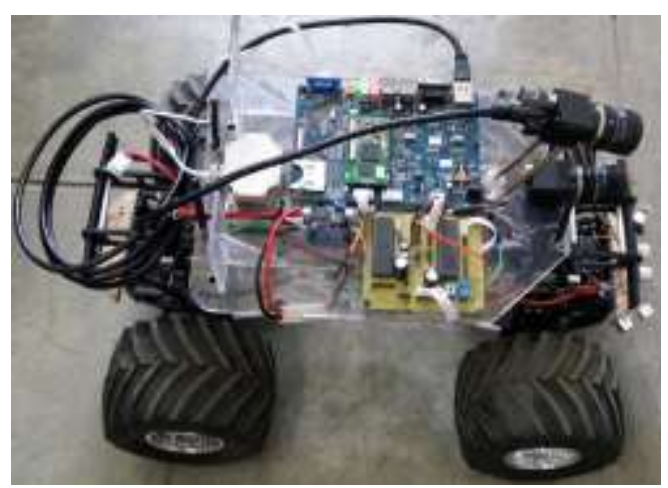

Figure 1. Test Vehicle

\section{Research on Data Fusion Algorithm}

In order to complete the autonomous operation under complex, dynamic and uncertain environment, a model vehicle is usually equipped with many kinds of sensors, such as CCD camera, ultrasonic sensor, gyroscope, and etc. Multi-sensors information fusion [37] involves integration of environmental information provided by multiple sensors of different types and integration of the external environment features to better detect information and identification degree of the environment. In this paper, the vehicle perceives environmental information accurately in multi feature information fusion method of target recognition in two-stage joint based on neural network and D-S evidence theory for intelligent vehicle navigation in unknown environment and uncertainty of sensor information. Firstly, the feature information is extracted from the environmental area by neural network, and the recognition results of multiple neural networks are fused with D-S evidence theory. This method makes use of multi feature information of the target to reduce errors of identification probability. And the two-stage joint information fusion model is of favorable scalability, and changes feature fusion information source conveniently and flexibly according to different objects and situation. The classification and dimension reduction method of input vectors greatly simplify the network structure and improve the generalization ability of network, but they produce several network identification results for further comprehensive treatment afterwards. Based on the design, a multi feature fusion recognition model of two-stage for neural network and D-S evidence theory is constructed, as shown in Figure 2. 


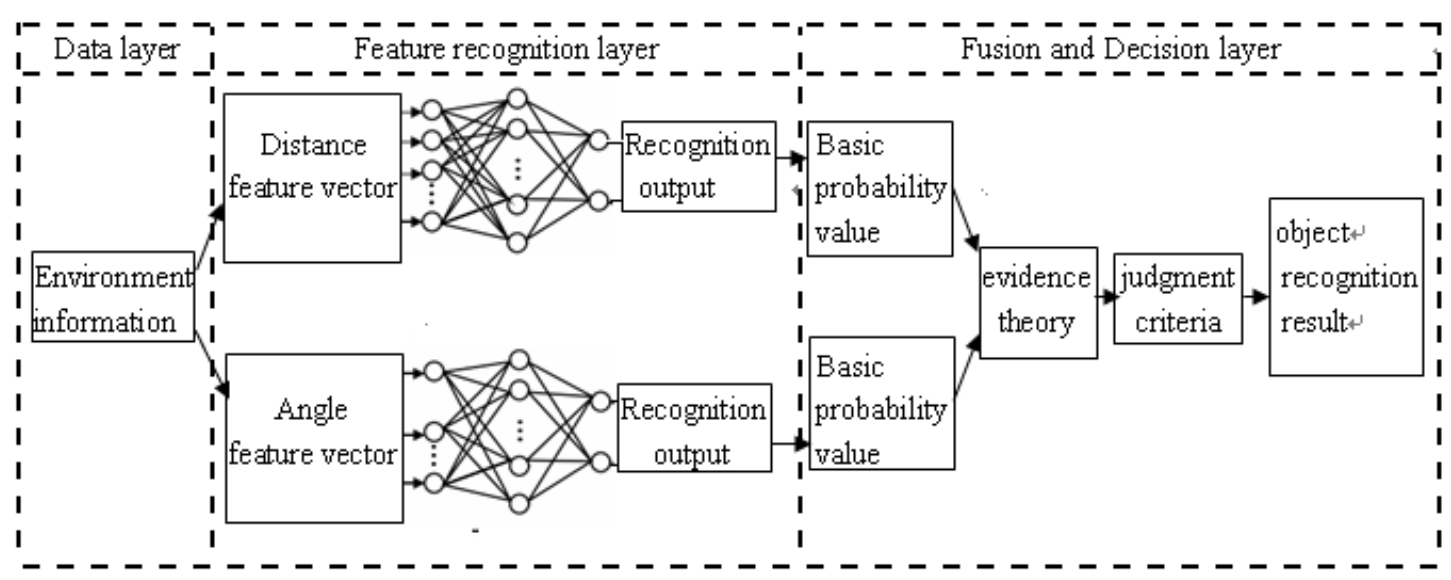

Figure 2. Information Fusion Model of Neural Network and D-S evidence Theory

In Figure 2, the information fusion model of multi features consists of three layers of the data, the feature recognition and the fusion decision-making. Information of the data layer from image data of the regional environment is achieved into two categories of distance and angle with different methods of characteristics and described by a set of feature vectors; feature recognition layer establishes the multi-layer perception network on each feature category to implement target recognition; fusion decision-making layer adopts D-S evidence theory to multi-layer perception network output for decision fusion, and turns the result into recognition results of the whole system.

\subsection{Data Fusion based on Neural Network}

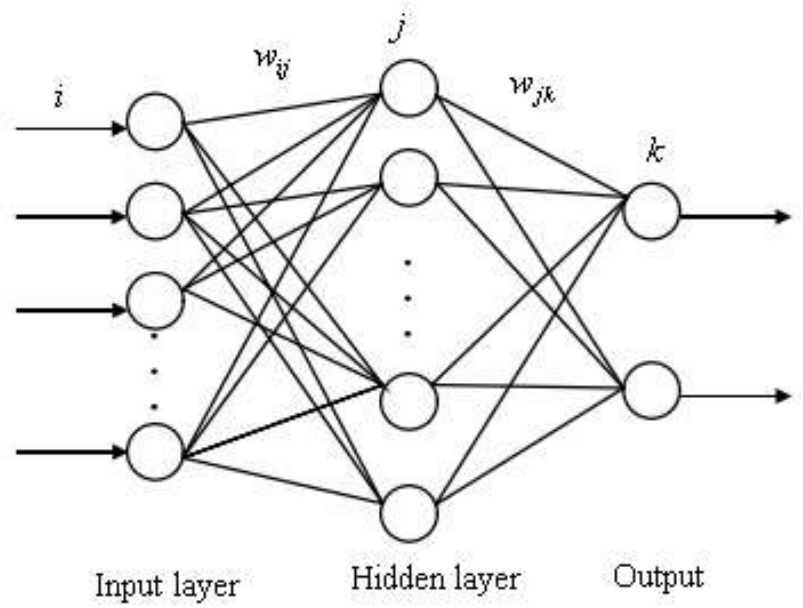

Figure 3. The Neural Network Model

Neural network [8] is a multi-layer processing system, including input layer, hidden layer and output layer, where implied layer can be one layer or multi-layer, and the typical three layer perception network structure is shown in Figure 3. In Figure, input layer is for the feature vector for recognition; hidden layer is for the calculation module of data transmission, and correction is the middle layer connecting the input layer to the output layer; and output layer is for the judgment results of preset categories in different feature information of target recognition. The neural network algorithm mainly includes the network training stage and the data testing stage. The network training uses the back spreading algorithm [9], which composes of the forward data transmission and back 
spreading of the error. In forward transmission process, a feature information of the feature vector acts as input layer of the entrance, through the hidden layer for computing and processing to the output layer as target verification results; in the back spreading process, judgment results of the output layer are compared with desired outcomes preset. If error exceeds the allowable range, the error signal from the output layer back spreads layer by layer with adjustment of each layer connection weights, and the revised network enables output judgment result gradually close to the preset expected results. After the forward transmission and the back spreading iteration, output of the network is converged to meet requirement of the expectations preset.

Its input-output relationship can be expressed as

$$
y=g\left(\sum_{i=1}^{n} k_{i} u_{i}-\theta\right)
$$

Where, $u_{1}, u_{2}, \cdots, u_{n}$ are the input signals, from the external environment or other neurons; $k_{1}, k_{2}, \cdots, k_{n}$ are the connection weights of each input; $\theta$ is threshold; function $\mathrm{g}$ is a transfer function $R \rightarrow R$, also known as activation function to indicate the neuron output.

Where, if $y_{j}$ is output of neuron $\mathrm{j}, x_{i}$ is the input of neuron $\mathrm{i}$ to $\mathrm{j} ; \eta$ the detecting rate parameter $w_{i j}$, connection weights between neuron $\mathrm{i}$ and neuron $\mathrm{j} ; \Delta w_{i j}$ corrected value of the connection weights $w_{i j}$, namely $w_{i j}(n+1)=w_{i j}(n)+\Delta w_{i j}$.

The minimum average variance between the defined output value and the desired value is

$$
E=\frac{1}{2}\left(d_{j}-y_{j}\right)^{2}=\frac{1}{2}\left(d_{j}-g\left(\sum_{k} w_{k j} k_{k}\right)\right)^{2}
$$

The learning rule $\delta$ requires minimum average variance by the right coefficient, and then

$$
\frac{\partial E}{\partial w_{i j}}=-\left(d_{j}-y_{j}\right) g^{\prime}\left(\sum_{k} w_{k j} x_{k}\right) x_{i}
$$

Therefore, the weight coefficient changes in the negative gradient direction, i.e.

$$
\Delta w_{i j}=\eta\left(d_{j}-y_{j}\right) g^{\prime}\left(\sum_{k} w_{k j} x_{k}\right) x_{i}
$$

As shown in Figure 3, $\mathrm{i}$ is a node of input layer, $\mathrm{j}$ is a node of hidden layer; $\mathrm{k}$ is a node of output layer, $d_{k}$ for the network's expected output, $y_{k}$ for actual output of the network. The error function of the network is defined as the average variance.

$$
E=\frac{1}{2} \sum_{k}\left(d_{k}-y_{k}\right)^{2}
$$

Sigmoid function acts as the activation function for nodes of the hidden layer and the output layer, i.e.

By BP algorithm, learning rule of the weight in each layer is as follows

$$
E=\frac{1}{2} \sum_{j}\left(Y_{i}-d_{i}\right)^{2}
$$

Where, $Y_{i}$ refers to the actual output of the network, $d_{i}$ is the expected output of the network.

The common activation function consists of those of the threshold, the partition and the Sigmoid, i.e. 
$g(x)=[1+\exp (-x)]^{-1}$

By BP algorithm, learning rule of the weight in each layer is as follows:

Layers of hidden and output, i.e.

$w_{j k}(t+1)=w_{j k}(t)+\eta \delta_{k} x_{j}^{\prime}, \delta_{k}=y_{k}\left(1-y_{k}\right)\left(d_{k}-y_{k}\right)$

Layers of input and hidden, i.e.

$w_{i j}(t+1)=w_{i j}(t)+\eta \delta_{j} x_{i}, \delta_{j}=x_{j}^{\prime}\left(1-x_{j}^{\prime}\right) \sum_{k} \delta_{k} w_{j k}$

Where, $\eta$ is the learning rate, normally $0<\eta<1 ; \delta_{k}, \delta_{j}$ are the corrected value; $x_{j}^{\prime}$ is the output at node $\mathrm{j}$ of the hidden layer, i.e.

$$
x_{j}^{\prime}=f\left(u_{j}\right)=\frac{1}{1+e^{-u j}}=\frac{1}{1+e^{-\left(\sum w_{i j} x_{i}-\theta_{j}\right)}}
$$

Node number of the hidden layer plays the decisive role for network calculation precision and convergence speed. There is no clear certain method to identify number of neuron nodes in theory; in most cases, it is obtained through a lot of experience. An empirical formula is used to make sure the number of nodes in the hidden layer through several experimental analyses in this paper.

$$
N=\left(N_{1}+N_{2}\right)^{1 / 2}+\beta
$$

Where, $\mathrm{N}$ is number of neurons in the hidden layer; $\mathrm{N}_{1}$ is number of neurons in the input layer, $\mathrm{N}_{2}$ is number of neurons in the output layer; $\beta$ is the adjustment parameter, $1 \leq \beta \leq 10$.

\subsection{Data Fusion based on D-S Evidence Theory}

The information fusion based on D-S evidence theory [10-12] acts as the decisive fusion for identification results of the multi-layer perception network to form the final decision of target attribute. D-S evidence theory fusion process is shown in Figure 4. When D-S evidence theory is used in the decisive fusion, the data information obtained from the multi-layer perception network is the evidence in the theory, where the trust degree can be reallocated to the information. Reasoning of D-S uncertainty evidence strengthens trust degree of the obstacle region, and weakens trust degree of the empty region, which achieve accurate environment information.

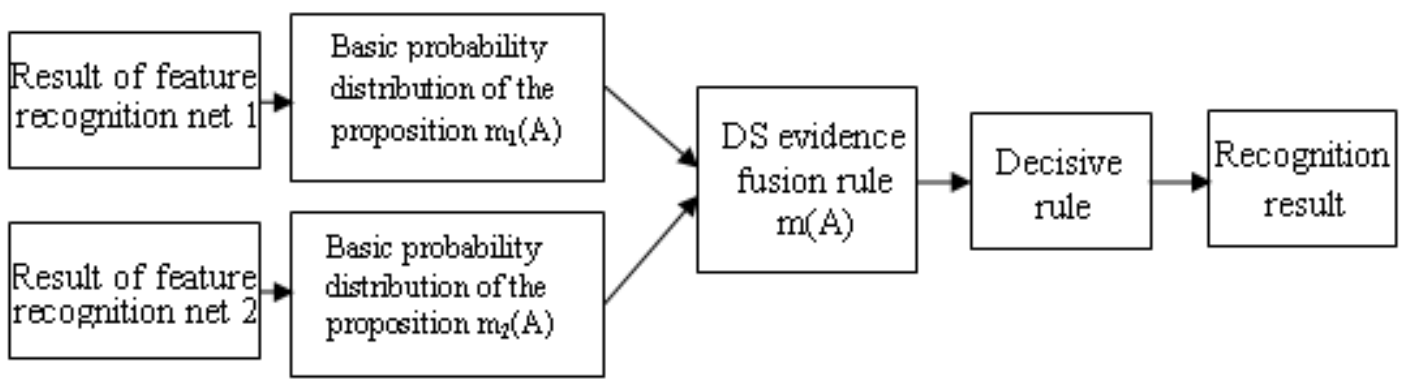

Figure 4. Fusion Process Based on D-S Evidence Theory

Intelligent vehicle navigation does not require complete feature recognition of obstacles. Thus, it is possible to define framework of the recognition $\Theta=\{\mathrm{E}, \mathrm{O}\}$, where, $\mathrm{E}$ means no obstacle, $\mathrm{O}$ means a obstacle. The power set of $\Theta$ is $2^{\Theta}=\{\Phi, E, O,\{E, O\}\}$, and correspondingly the basic function definitions of probability assignment is $\mathrm{m}$. For any 


$$
\begin{aligned}
& \text { region of space in fuzzy partition, } m(\Phi)=0 \quad, \quad \text { and } \\
& \sum_{A \subseteq 2^{\Theta}} m(A)=m(\Phi)+m(E)+m(\{O, E\})=1
\end{aligned}
$$

The output value of neural network is set at $\left(\mathrm{y}_{1}, \mathrm{y}_{2}\right)$, with $(1,0)$ as no obstacle and $(0,1)$ as obstacle, so the basic function of probability assignment is constructed in experiments.

For $\left|y_{1}\right|+\left|y_{2}\right| \leq 1.5$, the basic function of probability assignment is

$$
\left\{\begin{array}{c}
m(E)=\frac{\left|y_{1}\right|}{\left|y_{1}\right|+\left|y_{2}\right|} \\
m(O)=\frac{\left|y_{2}\right|}{\left|y_{1}\right|+\left|y_{2}\right|} \\
m(\{E, O\})=0 \\
m(\Phi)=0
\end{array}\right.
$$

For $\left|y_{1}\right|+\left|y_{2}\right|>1.5$, the basic function of probability assignment is

$$
\left\{\begin{array}{l}
m(E)=0 \\
m(O)=0 \\
m(\{E, O\})=1 \\
m(\Phi)=0
\end{array}\right.
$$

Fusion law of evidence

Rule of information fusion for multiple sensors is $m=m_{1} \oplus m_{2} \oplus \cdots m_{n}$, i.e.

$$
\begin{aligned}
& m(A)=K^{-1} \sum_{\cap A_{i}=A} \bigcap_{1 \leq i \leq n} m\left(A_{i}\right) \quad, A \neq \Phi \\
& K=1-\sum_{\cap A_{i}=\Phi} \bigcap_{1 \leq i \leq n} m\left(A_{i}\right)
\end{aligned}
$$

Where, $m\left(A_{1}\right), m\left(A_{2}\right), \cdots, m\left(A_{n}\right)$ indicates the basic probability assignment from the $\mathrm{n}$ multi-layer perception network, and $\mathrm{m}(\mathrm{A})$ indicates the basic probability assignment after the fusion.

Multi feature information fusion model identification in the evidence theory and evidence combination body how to fusion decision-making is directly related to the accuracy of the target identification, and in D-S evidence theory is not explicitly given specific fusion decision criteria. According to the characteristics of the intelligent vehicle navigation system, the decision formula of target recognition is set up in experiments.

$$
\left\{\begin{array}{l}
m(E)-m(O)>\varepsilon_{1} \\
m(E)>m(E, O) \\
m(\{E, O\})<\varepsilon_{2}
\end{array}\right.
$$

Where, $\varepsilon_{1}$ and $\varepsilon_{2}$ are preset thresholds. If the function is true, $A_{1}$ is the judgment, or else, the judgment is $A_{2}$.

\section{Road Planning}

Autonomous driving involves the core technology and fundamental purpose of intelligent vehicles. Road planning bridges up information perception and vehicle control and the basis for automatic driving, which is researched mainly based on road planning with uncertain multi-sensor information. Its basic idea is to use a lot of sensing device to 
sense the external environment by the real-time image data analysis and processing of information. The results will be transformed into running parameters and thus rely on executive control mechanism to determine appropriate speed and path and to realize autonomous driving function [13]. The difficulty lies in uncertainty and complexity of the road environment, and the contradiction between intelligent vehicle control and rapidity and real-time operation.

\section{Study on Immune Control Algorithm}

In design of intelligent vehicle system, steering control has a direct impact on performance of the vehicle. The conventional PID control algorithm is not so ideal for continuous deflection continuity of the front wheels in the process[14]. Immune controller solves the contradiction between the rapidity and the steady error of the system. Biological immune system dependent on T cells in different stages of immune promotes or inhibits the adjustment to ensure rapid response and maintain sufficient stability of the immune system in all antigen invasion, so as to maintain homeostasis of the organism. This paper proposes a new method of artificial immune control with the mechanism of company immunization of $\mathrm{T}$ cells and $\mathrm{B}$ cells in biological specific immunity as reference, and applies the method to control of the intelligent vehicle.

Based on the immune feedback rules and the conventional PID control, in Matlab/ simulink and Carsim environment, a self-adjusting immune feedback controller of PID (as shown in Figure 5 below) is designed and used to control the intelligent vehicle steering system with nonlinear disturbance.

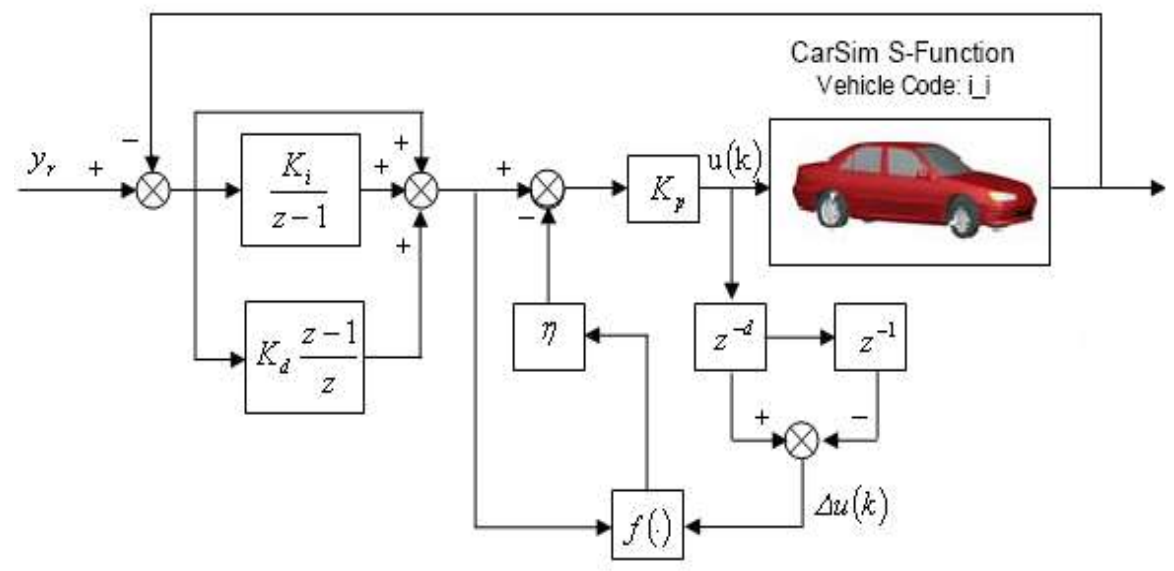

Figure 5. PID Type Self-regulatory Immune Response Controller

Where, $\mathrm{Kp}, \mathrm{Ki}$ and $\mathrm{Kd}$ are gain parameters, integral action parameters, and differential motion parameters; $\mathrm{Z}$ is the output of zero order. With output of PID controller as the external quality, the IMF controller is as follows:

$$
u(k)=K_{p}\{1-\eta f[\Delta u(k)]\}\left(1+\frac{k_{i}}{z-1}+K_{d} \frac{z-1}{z}\right) e(k)
$$

Where, the parameters of the artificial immune controller are selected as follows: the concentration of antibody $a, 5$; the delay parameter $\mathrm{d}, 1$; the immune parameter $\mathrm{Kp}, 1.1$; the inhibition parameter $\eta, 0.2$. Intelligent vehicle parameters are as follows: quality $\mathrm{m}$, $1300 \mathrm{~kg}$; yaw inertia Iz, 2550kg. m2; tread d, 1.50m; wheelbase $\mathrm{L}, 2.50 \mathrm{~m}$; distance from centroid to front axle lf, $0.95 \mathrm{~m}$; distance from centroid to the rear axle $1 \mathrm{r}, 155 \mathrm{~m}$; measuring partial stiffness of front wheel $\mathrm{k} 1,35700 \mathrm{~N} / \mathrm{rad}$; measuring partial stiffness of rear wheel k2, 43030N/rad. 


\section{Simulation Experiment}

Simulation experiments of double lane change are carried out according to the design of immune control, for which test site of the double lane change is set at ISO/3888 in Technical Reports and GB6323-86 standard, as shown in Figure 6.

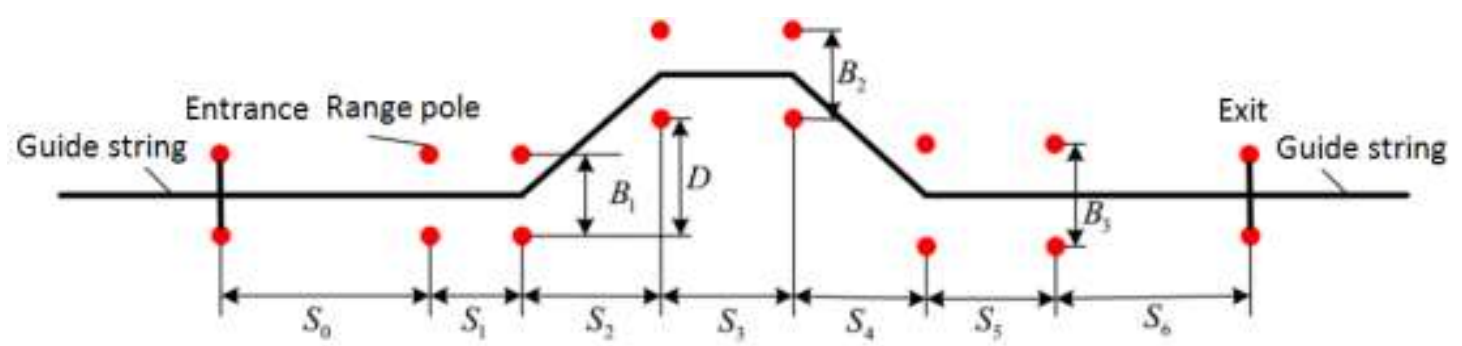

Figure 6. Simulation Test Road for Double Lane Change

Where, $S_{0}=50 \mathrm{~m} ; S_{1}=15 \mathrm{~m} ; S_{2}=30 \mathrm{~m} ; S_{3}=S_{4}=25 \mathrm{~m}, S_{5}=30 \mathrm{~m} ; S_{6}=50 \mathrm{~m}$; distance of lane change $\mathrm{D}, 3.5 \mathrm{~m}$; b, vehicle width; benchmark width $B_{1}, 1.1 \mathrm{~b}+0.25 \mathrm{~m}$; benchmark width $B_{2}, 1.2 \mathrm{~b}+0.25 \mathrm{~m}$; pole width, $1.3 \mathrm{~b}+0.25 \mathrm{~m}$.

Intelligent vehicle tracking is shown in Figure 7, and lateral acceleration of the smart car is in Figure 8.

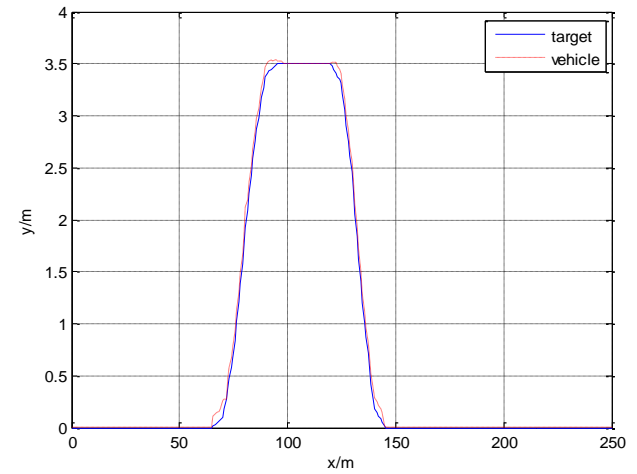

Figure 7. Lateral Offset from Design Road

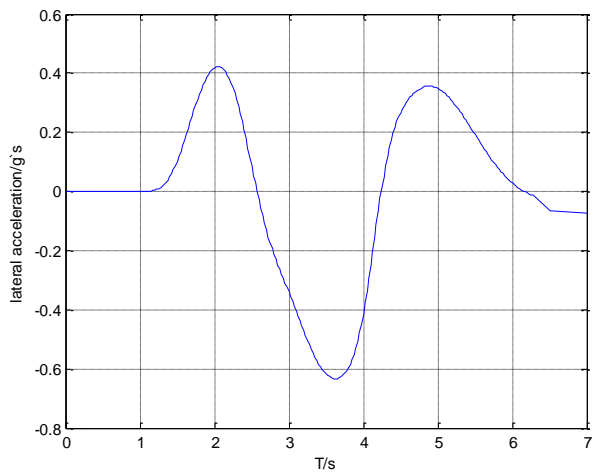

Figure 8. Lateral Acceleration of The Smart Car

In Figure 7, the intelligent vehicle trajectory and the desired trajectory are basically the same, indicating that design of the immune controller tracking is satisfactory, error control for lateral tracking is within scope of the provisions, and high control precision is achieved. As shown in Figure 8, lateral acceleration of the intelligent vehicle along the whole process of double lane change of test simulation is basically controlled within the range $\pm 6 \mathrm{~m} / \mathrm{s}^{2}$, which ensures comfort and stationarity of the intelligent vehicle.

Side slip angle of tire is shown in Figure 9, and lateral inclination angle of the smart car is in Figure 10. 


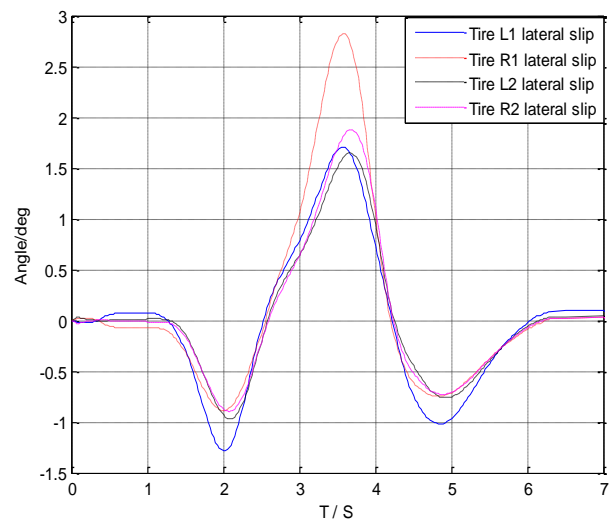

Figure 9. Lateral Slip Angle of Tire

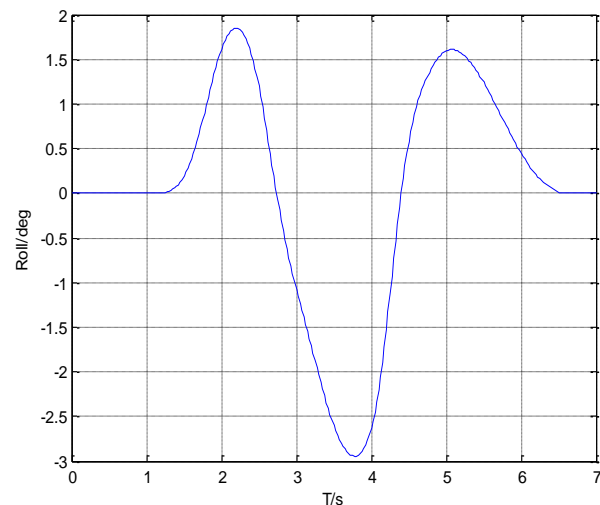

Figure 10 .Lateral Inclination Angle of the Intelligent Vehicle

In Figure 9, lateral slip angle of tire is controlled in $\pm 3^{\circ}$, and angle tracking error of the visible controller is fit. Lateral inclination angle, an important parameter for rollover risk of the intelligent vehicle, is controlled within $-3^{\circ} \sim 2^{\circ}$, which shows that the risk of vehicle rollover is low.

Steering wheel angle of the intelligent vehicle is shown in Figure 11, and vertical load of the front and rear wheels is in Figure 12.

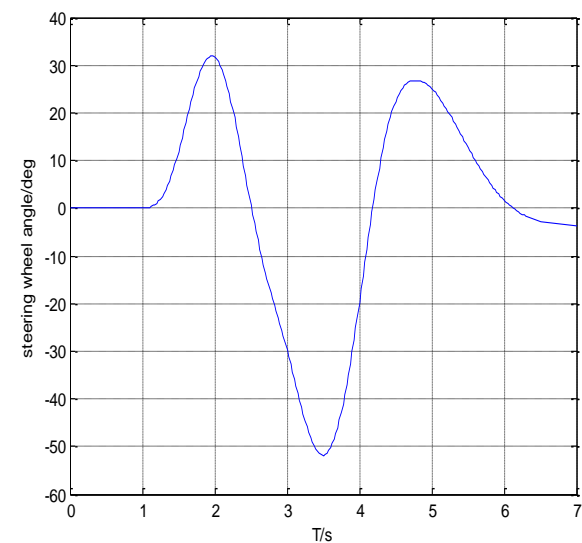

Figure 11. Steering Wheel Angle

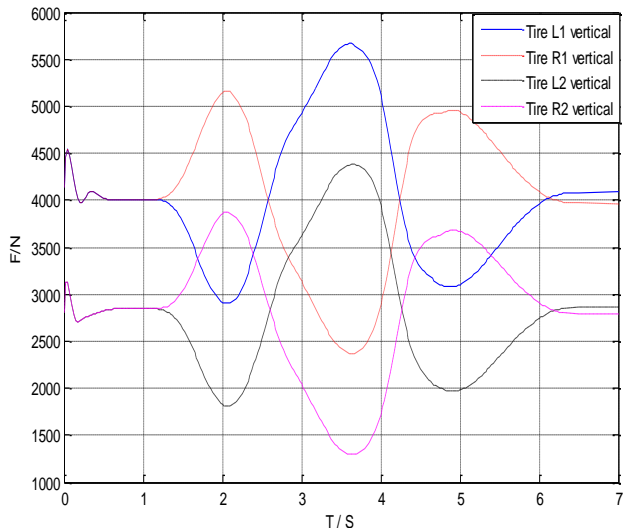

Figure 12. Vertical Load of the Front and Rear Wheels

In Figure 11 and Figure 12, vertical loads of the front and rear wheels, an important parameter for side slip risk of intelligent vehicle, are in reasonable ranges, which shows that the side slip risk is relatively low during the whole process of double lane change test simulation.

\section{Conclusion}

Intelligent vehicle is one of the key technologies in the intelligent transportation system and an integrated carrier of many high technologies. Simulation of miniature intelligent vehicle is an effective way to realize the development of intelligent vehicle. Practice has proved that research and design of miniature intelligent vehicle have realized basically the desired target function and task of research and development preset. The algorithm used for miniature car in laboratory environment plays an important role in acquiring advantages of multi-sensor system for intelligent vehicle, to obtain useful environmental information, to achieve autonomous driving control of the intelligent vehicle, and to 
promote intelligent vehicle development process. Research and test of miniature intelligent vehicle has succeeded initially, but there are still many defects and problems that need constant breakthrough and perfection. For example, reliability of the machine vision, stability of the control system and reliability of the whole vehicle still face a lot of problems, which need to be further solved.

\section{Acknowledgments}

This work is partially supported by Scientific and Technological Project under Grant 20130321005-04 and 20140311027-02. The authors also gratefully acknowledge the helpful comments and suggestions of the reviewers, which have improved the presentation.

\section{References}

[1] H. Chen, X. Gong, Y.F. Hu, Q.F. Liu, B.Z. Gao and H.Y. Guo, "Automotive Control:the State of the Art and Perspective", Acta Automatica Sinica, vol.39, no.4, (2013),pp.322-346.

[2] R. Bishop, "Intelligent vehicle applications worldwide", IEEE Intelligent Systems and Their Applications, vol.15, no.1, (2000), pp.78-81.

[3] B.C. Li, J. Huang and S.H. Gao, "Information Fusion Technology and Application, National Defense Industry Press", Beijing, (2010).

[4] M. Hamid, I. Dan and B. Jerome, "A Pervasive Multi-sensor Data Fusion for Smart Home Healthcare Monitoring", IEEE International Conference on Fuzzy Systems, Taipei, Taiwan, (2011).

[5] Y.T. Lan and J.Y. Huang, "A study of intelligent vehicle motion control system based on multi-sensor information fusion", 26th Chinese Control and Decision Conference, Changsha, China, (2014).

[6] W.Q. Wang, Y.L. Yang and C.J. Yang, "A data fusion algorithm based on evidence theory", Control and Decision, vol.28, no.9, (2013), pp.1427-1430.

[7] W.W. Chen, T.B. Wang, J. Jiao, M.L. Wang and J.E. Wang, "Design and Application for Navigation Multi-agent of Intelligent Vehicle Based on Information Fusion", Transactions of the Chinese Society for Agricultural Machinery, vol.42, no,6, (2011), pp.1-5.

[8] W. Li , S.C. Li and D. Wang, "Risk evaluation of the wind power project investment based on BP neural networks", Advanced Materials Research, vol.108, no 1, (2010), pp.256-261.

[9] D. Liu, Y.H. Tang, H. Yong, J. Wang and X.L Liu, "PID Control Algorithm Based on Improved BP Neural Network", Ordnance Industry Automation, vol29, no.3, (2010), pp.28-32

[10] C.Q. Tian and B.J. Yang, "A D-S evidence theory based fuzzy trust model in file-sharing P2P networks", Peer-to-Peer Networking and Applications, vol.7, no 4, (2014), pp.332-345.

[11] R.G. Zuo and Y.L. Cao, "Matting image based on D-S evidence theory", IEEE Conference Anthology, China, (2013).

[12] B. F. Chen and C. Ji, "Multisensor information fusion of pulsed GTAW based on improved D-S evidence theory", International Journal of Advanced Manufacturing Technology, vol.71, no 7, (2014), pp.91-99.

[13] A.F. Zhang, Y.C. Xu and J.F. Liu and J. Liu, "Design of Intelligent Vehicle Control System of Selfdirected", 24th Chinese Control and Decision Conference, Taiyuan, China, (2012).

[14] J.G. Bu, Y.C. Xu, S.Q. Dai and T. Han, "Neural Network Steering Control Algorithm for Autonomous Navigation of Vehicle", Journal of Academy of Armored Force Engineering, vol.21, no.4, (2007), pp.66-71.

\section{Authors}

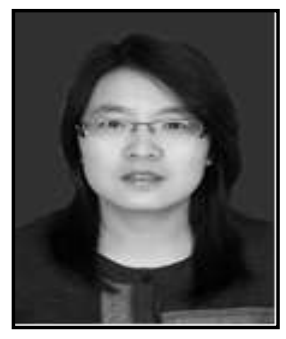

Yanting Lan, she is a Lecturer and $\mathrm{PhD}$, from College of Computer Science and Control Engineering, North University of China, major in application of data fusion and intelligent control Address: College of Computer Science and Control Engineering, North University of China, P.C. 030051 Email: lytcyb@foxmail.com 


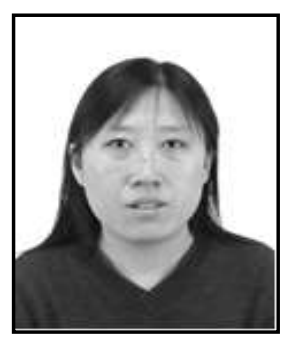

Jinying Huang, she is a Professor and doctor supervisor, major in Analysis and control of structure dynamics and intelligent vehicle Address: College of Mechanical and Power Engineering, North University of China, Taiyuan, P.C. 030051, Email : jyhuang@nuc.edu.cn.

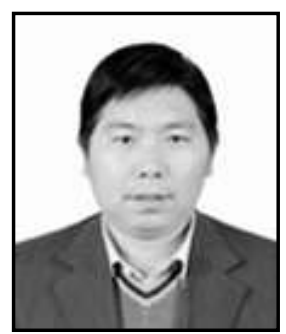

Xiaodong Chen, College of Agriculture and Biotechnology, China Agricultural University /Key Laboratory of farming system, Ministry of agriculture, $\mathrm{PhD}$, major in application of agricultural information, intelligent agriculture application. Address: College of Agriculture and Biotechnology, China Agricultural University, Beijing P.C. 100193。Email: xiaodong136@foxmail.com. 
International Journal of Control and Automation

Vol. 10, No. 5 (2017) 